\title{
Budgeting and accounting of software cost: Part 1
}

\begin{abstract}
A. Seetharaman
is Associate Dean \& Chairman, Center for Multimedia Banking, Investment \& Accounting Center for Multimedia Banking, Investment \& Accounting, Faculty of Management, Multimedia University, Malaysia. He has published over 60 articles in international refereed publications and is a member of the Malaysian Account Research and Education Foundation.
\end{abstract}

\section{Senthilvelmurugan}

is a part-time lecturer in IT at the Faculty of Management, Multimedia University, Malaysia. He holds a Masters degree in information technology.

\section{T. Subramanian}

is a lecturer in IT at the Faculty of Management, Multimedia University, Malaysia. He holds Masters degrees in computer applications, business administration and economics.

Keywords: costing systems, costing models, function point analysis, software development lifecycle

Abstract Many organizations today invest hugely in developing and launching information systems. The costs include labor-related consultation, software development and design fees, as well as software and hardware expenditures. However, with the subjective nature of software development, the estimation of software cost is usually inaccurate. Such organizations generally consult a software vendor to deliver information systems based on a set of specific user requirements. Deterministic approaches to software development cost are often miscalculated, however, and the end result is usually a higher cost for the customer. The scope of this study is limited to the fundamentals of software cost estimation approaches and how the results of these cost models are used to determine software cost. This paper is not a software engineering model. It focuses on only a subset of the software development lifecycle (SDLC) pertaining to the estimation of software size and cost. This paper highlights the issues determining the size of software and how they relate to the accounting and budgeting of software cost through the estimation of various software metrics to empirically predict the cost involved before starting development. The paper also discusses how project and software costs are derived from the results obtained from the cost metrics. For this purpose, a practical six-step approach is derived and presented. Finally, the limitations are discussed and a conclusion is given. The paper consists of two parts. The first part deals primarily with a survey of

Dr. A. Seetharaman Associate Dean \& Chairman

Center for Multimedia Banking, Investment \& Accounting,

Faculty of Management Multimedia University. 63100 Cyber Jaya,

Malaysia.

Tel: +60383125573

Email:

seetha@mmu.edu.my literature reviewing the trends in costing systems and constructive cost models. The second part of the paper will deal with selected case studies, models, discussion, analysis and findings.

\section{INTRODUCTION}

In the middle of the 20th century, hardware played a dominant role in determining the total cost of electronic data processing systems. It was an era of hardware-driven software. To meet the ever-mounting difficulties of the business world, there was a rapid growth of 
information technology and information systems leading to the age of softwaredriven hardware. This led to a situation in which a sizable percentage of the total cost of computer projects was being spent on software development. With the dawn of customer-driven software development, software costs further increased to around 80-90 per cent of the total cost of tailor-made IT projects.

Controlling and accounting for software costs are pressing problems for accountants and other managers, both in the systems and in the management accounting areas of the business. The main areas of software production activity are designed, developed, and monitored by specialist software houses. Software development cost control is, incontrovertibly, an important issue. Over- or under-estimation of the cost, late deliveries, poor quality, noncompatibility with hardware are examples of the seemingly intractable problems encountered in software development and maintenance activities leading to the software crisis.

Many authors have previously stressed the reuse of software for different purposes. Nevertheless, despite numerous papers in the areas of software architecture, development, and software reuse, authors have only addressed the economics of software. Understanding the determinants of software cost plays a critical role in software engineering management and control.

\section{RESEARCH PROBLEM}

Many companies are spending large sums of investments to develop and launch information systems. These costs include labor-related consultation and software development, plus design fees, as well as software and hardware expenditure. However, with the subjective nature of software development, the estimation of software cost is usually inaccurate. This problem is usually related to the following reasons:

- Thousands of software projects require significant investment from the customer without corresponding justification of the actual cost of the project itself. Many proposals and sales on software systems are made with inaccurate assumptions that lead to unnecessary high investment expenditure.

- Many research papers focus on issues pertaining to software cost metrics to determine the efforts required to deliver the information system. However, the information presented does not detail the accounting part of software costing.

- Many research papers are not intuitive enough to allow an inexperienced project manager to gauge the understanding into the complexities of software cost estimation using the available cost metrics.

- The problem of using jargon in the research papers is confusing and sometimes even conflicting. For example, some papers use the term cost models to denote the techniques used to determine the size of software; others use the term cost metrics.

- There is a lack of additional evidence to justify which cost estimation method to use for a particular situation. The papers in the literature focus on a set of industries only - this may bias the conclusion to those specific industries.

\section{Objectives}

The objectives of this research are:

- to explore the benefits of using cost metrics when determining software cost 
over the conventional "gut feeling" approach;

- to identify how labor effort (results obtained from using the cost metrics) can be turned into software cost;

- to provide a better understanding of the various cost metric approaches and which should be used in a particular scenario;

- to compare the different cost metric approaches.

\section{Scope}

The scope of this study is limited to the fundamentals of software cost estimation approaches and how the results of these cost models are used to determine software cost. It is necessary to emphasize that this paper is not a software engineering model. It focuses only on a subset on the software development lifecycle (SDLC)

(determining the project scope and cost during project proposal stage in the SDLC) pertaining to the estimation of software size and cost. However, the accounting process of how the software cost is derived from results obtained from the cost models will be discussed.

\section{RESEARCH METHODOLOGY}

The information presented in this paper was obtained from various sources of secondary data. The sources came mostly from the internet via search engines like Google, MetaSearch, Altavista and online searches from reputable online databases like ProQuest, Emerald Library and ACM Digital Library. The database sites were able to provide secondary data from sources

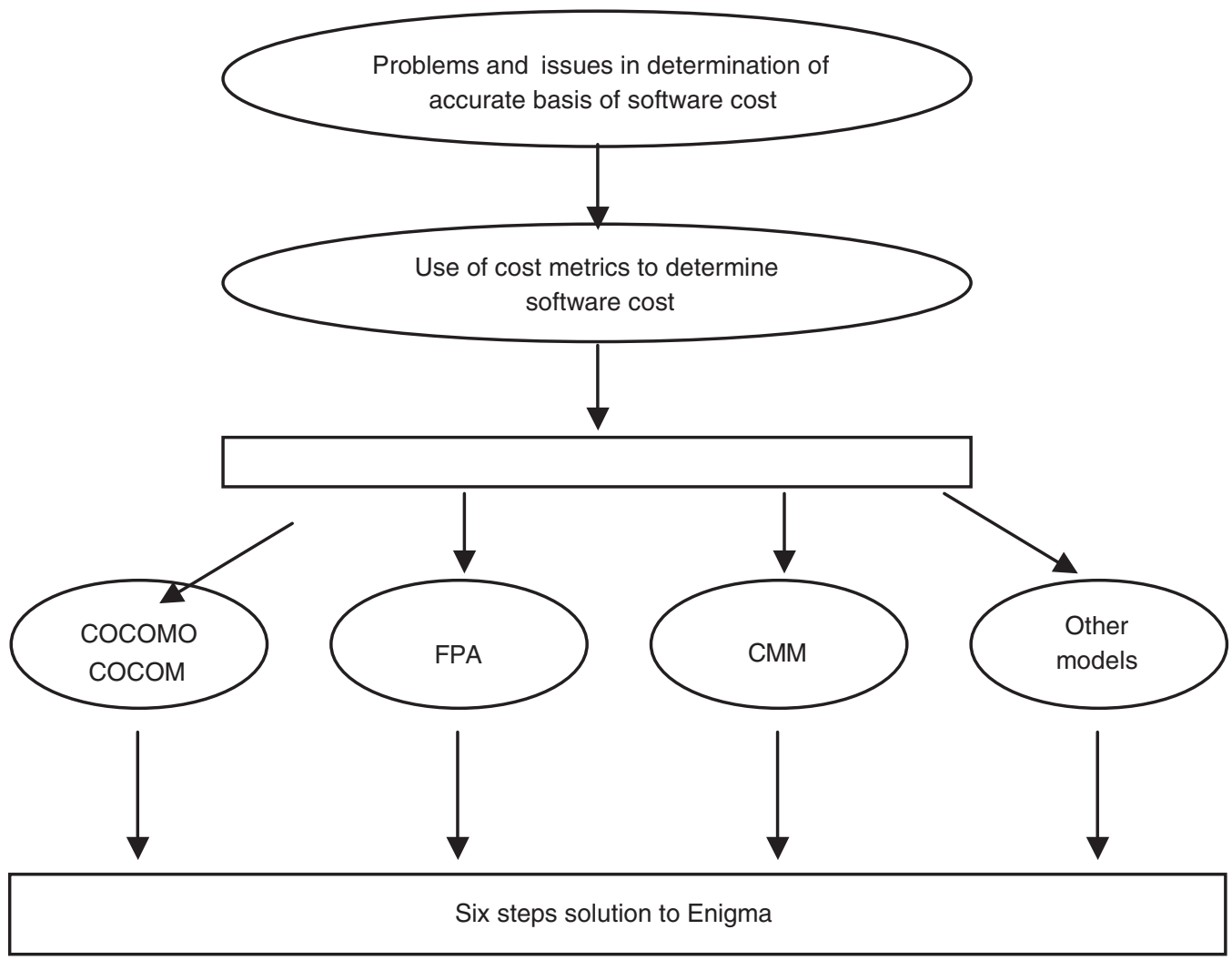

Figure 1: Research framework on determination of software cost 
such as journals and extracts from newspapers, books and magazines. Other references were also sought from compiled resources from the University library and books. More than 40 research papers were obtained pertaining to software costing.

The information obtained from these sources went through a rigorous process of short-listing. The authors vetted the information found within the research papers and selected more than 20 articles to be included into this paper. The authors decided to classify the research papers as depicted in Figure 1.

The next step was to analyze the information and to start preparing the document framework. Authoring commenced in tandem with analysis of the survey. The results from the surveys were processed to obtain a set of discussions, analysis and findings for this paper.

\section{SURVEY OF LITERATURE}

To facilitate meaningful discussion, it is necessary to place the literature into a framework for understanding and to serve as a "launch pad" for the contribution of prior work to the current paper. For this purpose, the survey of literature is grouped and presented under various headings namely, trends in costing systems, constructive cost model (COCOMO), function point analysis (FPA), and other models and case studies.

\section{Trends in costing systems}

Describing trends in costing software, Walker ${ }^{1}$ stated that cost estimation models in software engineering were introduced with the prevailing of computers in the mid-1960s. The cost estimation techniques were introduced to reduce the difficulty in estimating the size of software projects, with the hope of eliminating the "gut feeling approach" of estimating software cost. In the early 1960s, software engineering was based on craftsmanship, ${ }^{2}$ where the skills of the project team greatly contributed to the success of the project. Each of the projects had custom-built tools and processes in place and reusability of software codes was scarce.

Through the 1980s and 1990s, the software industry matured with the availability of more software cost models and methodologies. However, even with the abundance of such cost models, this era of software engineering still focused greatly on research and development ( $R \& D)$, hence the economies of software cost still depended on the creativity of the project team. $^{3}$

This situation in the software industry today has, however, changed. The software process is more driven towards a productive-centric approach where, with the availability of case tools and methodologies, the software project team can now focus on the real problem of building the information system and not on the engineering process itself.

The trends in software engineering are depicted in Figure 2. ${ }^{4}$ Over the years, it can be seen that budgeting the cost and deriving estimated efforts and timeline for software are becoming more predictive and accurate.

However, Walker has not detailed the reasons why software projects still fail due to running over budget and timeline. Even with the available tools, why are some projects successful while others fail to meet their objective? The various points of failure in software engineering should have been covered 


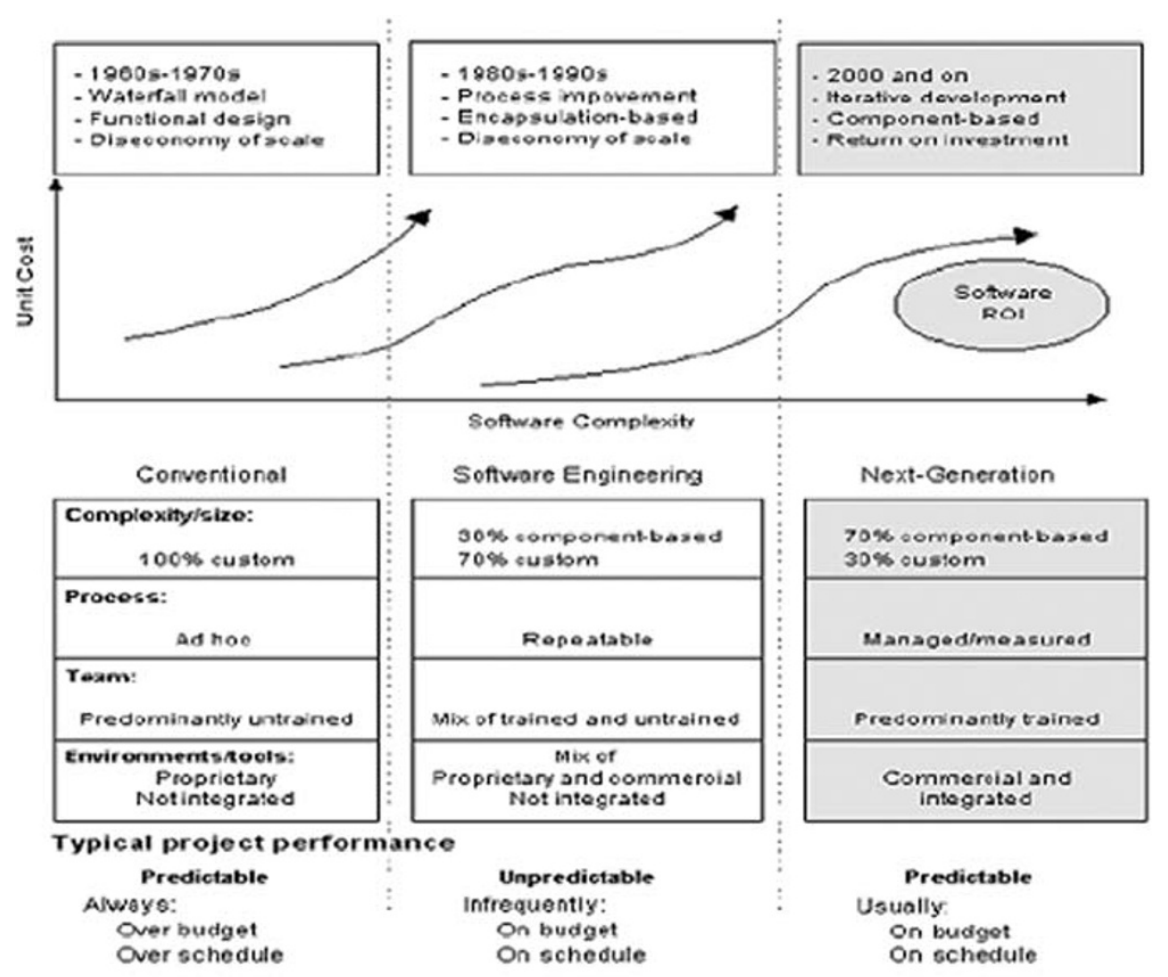

Figure 2: Trends in software economics

Source: Walker, R. (2001) "Improving software development economics Part 1: Current trends" (see ref. 1).

using modern cost estimation techniques, supplemented by effective measures to tackle them.

Chulani ${ }^{5}$ and Boehm et al. ${ }^{6}$ suggested that there are several ways to estimate the cost of software engineering. The various methods available suggest that there is no one way to estimate software development cost, and that it is a subjective matter.

Over the past several decades, there has been a growing number of software cost estimation techniques, parametric models, expertise techniques, dynamicbased models, models that utilize Bayesian techniques, and many more that face the challenges of ever-changing information technology. With the advent of Unified Modeling Language (UML), XML, and web services, such metric models require revision in order to sustain a level of accuracy in the real world.

Chulani suggested that although many techniques are available, no one technique is better than the rest. It is up to the project team to decide the best estimation approach to use and, sometimes, a combination of the estimation models is desirable to obtain the best result for a particular situation.

The authors, however, did not recognize that the estimation techniques used are strongly coupled to their environment, the project team and the nature of the software itself. These factors provide risks that are inherent during software development and can pose a threat to software completion.

Verner and Evanco ${ }^{7}$ identified the reasons why software projects fail due to under-budgeting and lack of resources. 
The authors surveyed the practice of software cost estimation in real business environments. Their research tended to combine the tangible and intangible cost factors that many other research papers failed to address.

Their survey found that only onethird of software projects involved the project manager during the initial software cost estimation. They found that predicting a realistic software cost estimation was necessary to the success of implementing the said software. The majority of the project managers were not involved in the initial software cost estimation.

The authors also stressed that identifying and allocating the right number of resources (programmers) to the project is key to its success or failure. This factor is particularly important in projects with an aggressive schedule. The authors found that the effect of adding staff (programmers) very late in the project schedule could be disastrous. Indeed, many projects failed because of failing to add staff earlier in the project development lifecycle.

A shocking discovery was that all the surveyed projects were over-estimated, which shows that optimism still prevailed during the software budgeting process. Almost all the projects were estimated with unclear requirements, leading to assumptions. The lack of proper risk management adds to the predicament of software failure (under budget, over schedule and lack of staff).

The quality of data samples was, however, a drawback to this study. None of the surveyed projects used the proper cost estimation methods discussed in this paper (COCOMO, function point etc). This did not reflect the ideology of software cost estimation theory, which suggests that with the proper cost estimation process, the chances of under-budgeting and staffing would be reduced greatly. Hence, Verner and Evanco might have provided a more robust conclusion had the data samples been segregated accordingly.

\section{Constructive cost models 1 and 2 (COCOMO)}

The original COCOMO cost estimation method was introduced due to the experience of increasing difficulties in estimating the cost of software.

However, as software matured to levels beyond those that COCOMO could support, Boehm derived a new metric model from the COCOMO model, and introduced COCOMO 2 in $1981 .^{8}$ The COCOMO 2 model is a hybrid of software sizing methods, ranging from object point analysis, function point analysis and source line of code and including source code reusability. The objectives of COCOMO were:

- to address the issues of modern software development and to pre-determine cost and schedule before the project has started;

- to collect data and statistics on current software cost so as to provide a set of data to empirically enhance and improve on the COCOMO model;

- to provide a quantitative analytical framework, tools and techniques for evaluating the software lifecycle to further improve the software lifecycle cost and schedule.

Boehm discussed the future trends of software engineering and how his cost model could be used to derive sizing and cost estimation for the software. He also 
discussed the differences between COCOMO and COCOMO 2, and the strategy and rationale behind the introduction of the COCOMO 2 model. Boehm showed empirically how gross effort was calculated from the components of COCOMO 2 (using object and function point analysis) and how the introduction of cost drivers affected the net effort of the software, which in return could be used to calculate software cost.

Boehm did not, however, mention that software cost also depends on a few intangible cost factors that can only be derived from past project experience, historical data and through observation of the project team's competency.

Intangible cost factors that can contribute to the increase in software cost include: skills and experiences of the project team members (project manager, system analyst, analyst programmers, programmers); delays due to unforeseen circumstances like change in governmental policies which directly affect the software system (payroll, HR management systems) etc; and the competency of the customer's project team in managing the project.

Abts et al. ${ }^{9}$ discussed another cost estimation model for software which is a derivation of the COCOMO model introduced by Boehm. The model, known as the constructive commercialoff-shelf (COCOTS) model can be used to estimate cost for small to mediumsized software projects with average complexity. However, it has its limitations in dealing with larger projects and other cost models like COCOMO 2 should be used instead.

The authors provided a module overview of COCOTS, describing the four sub-models:
- Assessment: the activity whereby COTS software products are vetted and selected as viable components for integration into a larger system.

- Tailoring: the activity whereby COTS software products are configured for use in a specific context.

- Glue code: the new code needed to get a COTS product integrated into a larger system.

- Volatility: system volatility effort (SVE) refers to that extra effort which occurs during the development of the larger application as a result of the use of COTS components in that system development.

It was found that the greatest COTS integration effort tends to be concentrated on glue code development. The authors suggested that this is due to the fact that the difficulties that must be addressed in glue code are often not apparent until the COTS integration process is well underway, making backtracking of designs and rework a necessity.

The authors ended the paper abruptly by stating that the total COTS integration effort was the sum of the four components above. There was no conclusion or summary in the research paper, which was a limitation as the reader would have to assume the conclusion of the authors.

Chulani et al. ${ }^{10}$ introduced the Bayesian analysis technique of calibrating software cost. Bayesian analysis uses inductive reasoning which is used in many scientific disciplines today. One distinctive feature of this approach is that it permits the investigator to use both sampled data and expert judgment in a logically consistent manner in making inferences. 
Bayesian analysis is applied on the COCOMO 21998 metrics $^{11}$ using two statistical approaches, the multiple regression approach and the Bayesian approach. Most cost estimation models, including COCOMO 21997 use a form of multiple regression approach, which prevents project managers from making good decisions from quality data. The Bayesian approach alleviates this problem by making use of expert judgment data with sampling information in a logically consistent manner.

The Bayesian approach incorporates a formal process of merging historical data with existing data. In other cost metrics, the ability to incorporate proper historical data is limited, hence the Bayesian approach processes data in a more competent way to provide project managers with an environment for better decision making in software projects.

Although the authors provided a thorough paper on the Bayesian approach, many assumptions were made pertaining to human factors in managing this approach. An inexperienced project manager might find this method a tedious and rigorous approach to estimating and controlling software cost. Hence, it would have been beneficial for the authors to provide a skill rating for this research paper so that junior project managers could meet the prerequisites of this paper before attempting to engage Bayesian analysis in estimating the cost of their software project.

\section{Function point analysis}

Roetzheim ${ }^{12}$ provided three excellent papers to discuss how function point analysis could be used in determining the size of software. The author covered the issues of calculating unadjusted software function points from user requirements, which can be used to estimate the number of lines of code, which in return is used to determine the estimated labor efforts required to complete the software project. The author refined the unadjusted function points by introducing cost drivers. The software effort estimation process was then further fine-tuned by introducing the concept of software component reusability.

Software reusability is a componentbased concept that can greatly reduce the time to develop software. In this context, software is developed from a library of components, akin to manufacturing a laptop computer. For example, the laptop manufacturers need only be concerned about designing the laptop around the existing peripherals like hard disk, monitor, touch pad, battery etc. They need not worry about designing and manufacturing the peripherals, and hence, this greatly improves their economies of scale.

The author did not, however, further elaborate on how the labor efforts could be used to determine the cost of the software; rather, he left the reader to gauge the software cost based on current accounting principles and practices.

Vickers provided an excellent, more thorough research paper on FPA. ${ }^{13}$ The paper addressed the fundamentals of why software measurement with numbers was far more accurate than the "gut feeling" approach. The author took the classified software metrics into two classifications: direct and indirect metrics. Direct metrics are cost factors that contribute directly to the overall cost of the project (eg cost, effort, speed etc) and indirect metrics, as the name 
suggests, involve cost factors that are usually indirect to the cost of the software (eg function, quality, complexity, efficiency etc).

The author then discussed the meaning of line of code (LOC) used in function point analysis and how it differs from one computer programming language to another. Vickers touched on the issue of productivity and how it can determine the cost of software, an issue which was omitted by Roetzheim. ${ }^{14}$ Productivity of the project team is an intangible issue that can only be measured by statistically calculating the maturity index (MI) and productivity index (PI) of the project team which can be a determining cost factor during the cost estimation process.

Vickers' paper went on to explain how function point analysis is used, albeit in a more technical way as compared with Roetzheim's paper. The author then discussed the benefits and problems faced by using FPA and how its limitations were overcome by the introduction of MKII Function Point Analysis (MKII FPA) pioneered by
Symons. ${ }^{15}$

Although Vickers' paper discussed FPA to a significant extent, its method did not allow for the problems of software development delays. Delays in software development (an inherent risk) cannot be controlled by determining metrics based on direct or indirect metrics. Hence, the paper assumed that software is handled by experienced project managers who can control this potential risk with professionalism to reduce its effect. Further, it adhered to the basic cost accounting elements, namely direct and indirect costs.

Symons wrote a paper on the reasons of decline for the usage of FPA in software cost estimation today. At the time of FPA's creation by Allan Albrecht of IBM, software was small to medium in terms of design complexity and size; hence FPA was widely used at that time. Modern software development, however, does not favor the old FPA, hence the author examined the reasons for this decline in using FPA. The author further examined the

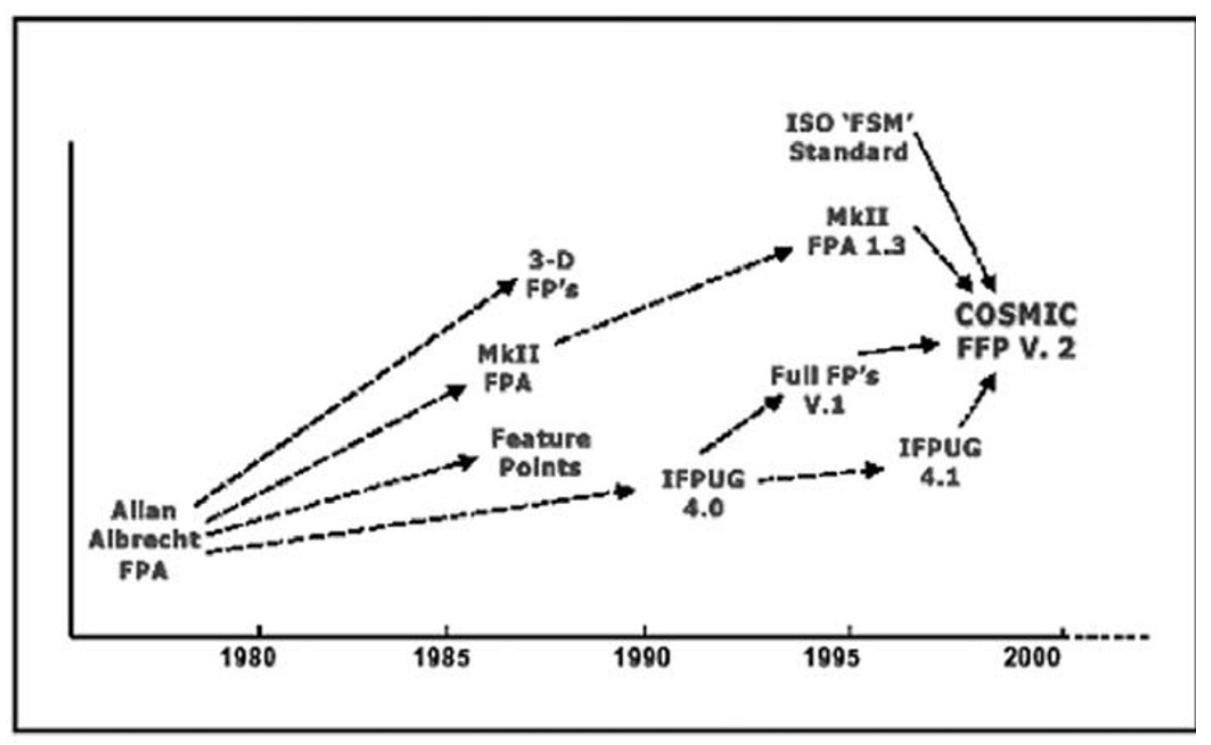

Figure 3: The evolution of function point analysis. 
evolution of FPA and through its progress, COSMIC FFP was derived from the International Function Point User Group (IFPUG). Figure 3 shows the evolution of function point analysis.

Symons provided the insight into the benefits of COSMIC FFP in modern software development, an area that the obsolete FPA did not. However, Symons' research covered only software metrics related to FPA. There are other software cost estimation techniques in the software industry and it would have been more comprehensive had Symons contributed a section in his paper to the comparison between these cost models.

Fabrizi's research paper presented a study on FPA software project measurement being utilized by Banca d'Italia, the Italian central bank. ${ }^{16}$

The author provided a time/effort comparison of past, present and future development methodologies adapted by the bank. In the past, the bank utilized a "waterfall" methodology to software development. Most projects were outsourced to third-party software vendors, contracted on a three-year agreement with software vendors. The bank currently still uses the "waterfall" model, but for the future, it is working towards a "back-bone with traffic lights" methodology and the outsourcing contract remains the same.

This paper was limited in that it only identified FPA within the context of the Italian bank and the research was limited to FPA. The author should have provided a general paper on software measurement with function point and from a wider perspective, including other industries.

Wu's research on FPA stressed the complexity of sizing software and determining its cost. ${ }^{17}$ The paper discussed the available methods for software size estimation, starting by explaining LOC, which is one of the oldest methods of software size estimation. How FPA was derived from LOC was then discussed, and explained.

The authors then surveyed 500 firms in Hong Kong to obtain information about software sizing, development tools, effort estimation and factors affecting software development time and activities. The data were collected and analyzed by the authors.

It was found out that 77.1 per cent of companies were using the LOC method and 22.9 per cent were using the FPA approach. This indicates that LOC was more favorable as it was easier to use than FPA. It was also found that the project manager's experience and user participation in the requirements specification stage were crucial to the implementation success of the project. Finally, it was found that FPA had more benefits than LOC, as LOC tends to be influenced by the type of programming language used. Hence, for a project that utilized multiple programming languages, this approach tends to be less feasible.

Although the research paper provided some commendable demographic results, it did not illustrate the effects of other cost drivers on FPA and LOC. The authors failed to identify the economic factor that can influence the total estimate of software which determines the final size of the software to deliver and the investment that has to be allocated.

The Armour et al. ${ }^{18}$ paper discussed managing the user requirements volatility which affects the total cost of the overall software project. The authors identified that, as the development of 
the project commences, new

requirements can be added by the customer. This will have a significant impact on the cost and timeline of the software project. In order to control this situation, the authors proposed a lifecycle estimation framework (LCEF) which consists of a number of estimating methods applied at key areas within the project lifecycle.

To determine the labor efforts needed to comply with the addition of new requirements, the size of the requirements are indicated by functions, which are akin to the function points found in FPA. The authors suggest that each requirement be assigned a set of function points to denote the size and complexity of the requirement. The requirements are grouped together and a regression analysis is performed. FPA is used to determine the number of function points for each requirement. Hence, the final software size can be determined based on the number of function points for each requirement.

The authors' research, however, did not state the size of the software projects used in the study. This can be a limitation in the research paper as complexity of software is directly proportional to software size increments.

\section{Other models}

Victor and Daily ${ }^{19}$ introduced another approach for determining software cost. This approach is known as the SPECTRE Model. The SPECTRE approach is akin to FPA except that it utilizes what is known as a construction point evaluation (CPE). CPE is different from FPA from the point of view of the target entities and fundamental objectives. The SPECTRE task estimation consists of three phases:
- Construction evaluation which focuses on two classes of functions, input/output and processing;

- Development effort calculation which focuses on identifying the features of processing logic like data restructuring, sorting, conditions, data retrieval, calculations as such; and

- Results display displays the result of the task estimate in two sections:

- Section 1 consists of actual estimates like LOC, development effort in days etc.

- Section 2 consists of additional items which are of value and interest like complexity rating of the software, experience rating and etc.

The SPECTRE model has a realistic prediction technique. Victor put his SPECTRE model through vigorous test cases to see the standard deviation of this model. It was found that an average standard deviation of 5.4 was discovered when comparing the estimated LOC with the actual results. Table 1 depicts this result.

Although the SPECTRE model offered good prediction, the authors

Table 1: The standard deviation between estimated and actual LOC derived from using the SPECTRE approach

\begin{tabular}{lcrl}
\hline $\begin{array}{l}\text { Test case } \\
\text { programs }\end{array}$ & $\begin{array}{l}\text { LOC } \\
\text { (estimated) }\end{array}$ & $\begin{array}{l}\text { LOC } \\
\text { (actual) }\end{array}$ & $\begin{array}{l}\text { Deviation } \\
\text { (\%) }\end{array}$ \\
\hline 1 & 1,287 & 1,338 & -3.9 \\
2 & 4,715 & 4,848 & -2.8 \\
3 & 5,684 & 5,695 & -4.7 \\
4 & 1,560 & 1,551 & +0.6 \\
5 & 11,280 & 10,776 & +4.6 \\
6 & 5,520 & 5,963 & -7.5 \\
7 & 540 & 634 & -14.8 \\
8 & 11,424 & 12,590 & -10.0 \\
9 & 2,277 & 2,342 & -2.8 \\
10 & 4,175 & 4,080 & +2.3 \\
\hline
\end{tabular}

Source: Victor \& Daily $(2001)^{22}$ 
failed to derive how the final software cost is determined. Again, like other cost models, the model focuses on labor efforts needed to deliver a software product based on the number of lines of code that need to be written.

Kokol et al. ${ }^{20}$ conducted research on the impact of using software metrics and evolutionary decision trees to control the quality of software. The aim of the research paper was to present the $\alpha$ metric and the usage of $\alpha$ in decision trees so as to produce a fault predictive metric.

First, the reliability and complexity of software metrics was discussed and its weaknesses were revealed. Then the authors described the physical background of communication, comparing a computer program and natural language texts. The methods of describing the correlation properties of texts were discussed and one such method was known as long range correlation (LRC). LRC can be used to quantify a software system's behavior and can allow for the recognition of similarities between systems. This induced the results required to allow the authors to show how software fault prediction using decision trees could be potentially identified.

This research was limited by the inability to identify the accounting aspects of producing software reliability and how this could in turn become a cost-saving factor within the environment of an organization.

Woodings and Bundell ${ }^{21}$ consolidated their efforts to discuss a framework for software metrics. Their paper defines a taxonomy of software metrics which is derived from the needs of users, developers and management.

The authors discussed the properties based on this classification, and introduced some rules (known as Metrel (Metric Relationship) rules) that were used to generate a new set of software metrics from an existing class. The authors claim that by using the Metrel rules, management, staff and customers would be able to view the success and process improvement efforts of software on one graph. This approach can be beneficial during meetings between the project team and the customer. In addition, the Metrel rules approach provides a check into the way software metrics are chosen and is employed as a supplement to the existing methods of metric definition and generation. Lastly, the Metrel rules are important to the modeling of the software development process by providing a mechanism for the expansion and transformation of metric models.

This paper was limited by the inability of the authors to show how the results of the Metrel rules are able to affect the accounting of software, which relates to how decisions are made within the business.

\section{NOTE}

Part 2 of this paper will be published in the next issue of the Journal.

\section{REFERENCES}

1. Walker, R. (2001) "Improving software development economics Part 1: Current trends," The Rational Edge, Available at: http://www.therationaledge.com/ content/apr_01/f_econ_wr.html.

2. Ibid.

3. Ibid.

4. Ibid.

5. Chulani, S. (2001) Software Development Cost Estimation Approaches, University of Southern California, Los Angeles, CA.

6. Boehm, B., Clark, B., Horowitz, E., Westland, C., Madachy, R., and Selby 
R. (1995) Cost Models For Future

Software Lifecycle Processes:

COCOMO 2.0, NEC Research

Institute, Available at: http://

citeseer.nj.nec.com/boehm95cost.html.

7. Verner, J. M. and Evanco, W. M. (2000)

State of the Practice of Effort Estimation in

Business Environments, European

Software Control and Metrics. Available

at: http://www.escom.co.uk/

conference2000/index.shtml.

8. See ref 5 above.

9. Abts, C., Boehm, B.W. and Bailey Clark, E. (2000) COCOTS: A COTS

Software Integration Cost Model - Model

Overview and Preliminary Data Findings,

Software Control and Metrics. Available

at: http://www.escom.co.uk/

conference2000/index.shtml.

10. Chulani, S., Boehm, B. and Steece, B. (2000) Calibrating Software Cost Models Using Bayesian Analysis, University of Southern California, Los Angeles, CA.

11. See ref 5 above.

12. Roetzheim, W. (2000) "Estimating software costs," Software Development Magazine Online, October. Available at: http://www.sdmagazine.com/print/ documentID = 11137; Roetzheim, W. (2000) "Project cost adjustments," Software Development Magazine Online, October. Available at: http:// www.sdmagazine.com/print/ documentID = 11124; Roetzheim, W. (2000) "Calculating for reuse," Software Development Magazine Online, December. Available at: http:// www.sdmagazine.com/print/ documentID $=11115$.

13. Vickers P. (2001) An Introduction to Function Point Analysis, School of Computing and Mathematical Sciences, Liverpool John Moores University, Liverpool, UK.
14. See ref 5 above.

15. Symons, C. (2001) Come Back Function Point Analysis (Modernised), European Conference on Software Measurement and ICT Control. Available at: http:// www.escom.co.uk/conference2001/ download.shtml.

16. Fabrizi, S. (2001) Software Project Measurement Using Function Point: A Continuous Improvement, European Software Control and Metrics. Available at: www.escom.co.uk/conference2001/ papers/fabrizi.pdf.

17. Wu, I.K. (2000) Using Function Point Analysis Method or Line of Code for Software Size Estimation?, European Software Control and Metrics. Available at: http://www.escom.co.uk/ conference2000/index.shtml.

18. Armour, F., Catherwood, B. and Beyers, C. (2001) "A framework managing requirements volatility using function points as currency," Proceedings of the ESCOM 2001, April 2001, London, pp. 295-303.

19. Victor, D. and Daily K. (2001) Software Estimating at the Task Level - the SPECTRE Approach, European Software Control and Metrics. Available at: http://www.escom.co.uk/ conference2001/download.shtml.

20. Kokol, P., Podgorelec, V. and Pighin, M. (2001) Using Software Metrics and Evolutionary Decision Trees for Software Quality Control, European Software Control and Metrics. Available at: http://www.escom.co.uk/ conference2001/download.shtml.

21. Woodings, T.L. and Bundell G.A. (2001) A Framework for Software Project Metrics, NEC Research Institute. Available at: http://citeseer.nj.nec.com/ 538619.html.

22. See ref 19 above. 